\title{
Meeting Abstracts of the 3rd International Workshop No-Cancer 2018 - understanding cancer cell biology to improve diagnosis and therapy
}

\author{
Novara, Italy; 28-30 Oct 2018; Published: 23 Jan 2019 \\ Correspondence to: Dr. Ciro Isidoro, Prof. Gianluca Gaidano, Università del Piemonte Orientale "A. Avogadro", Novara 28100, \\ Italy.E-mail: ciro.isidoro@med.uniupo.it; gianluca.gaidano@med.uniupo.it
}

\section{Editorial Note:}

Cancer remains a leading cause of death, despite the undeniable progress made in the last decades in the diagnosis and therapeutic treatments. Further improvements are only possible if we understand more in depth how cancer develops and metastasizes, and how it produces its damaging effects on the whole organisms, including immune-suppression and cachexia, that eventually lead to patient death. The goal of the International Workshop No-Cancer 2018 "understanding cancer cell biology to improve diagnosis and therapy" held in 28-30 Oct, in Novara (Italy) is precisely to share the foremost updated knowledge on the biology of cancer and the emerging technologies for early detection of cancer cells and the new "chemo-free" therapeutic options.

This is the third international Conference on Cancer organized under the auspices of the Università del Piemonte Orientale and other Academic and Cultural institutions, and ideally follows the two previous ones "Basic to Translational Medicine 2016: focus on Cancer" and No-Cancer 2017 "From Cancerogenesis to Therapy: new paradigms, new opportunities", held in Novara. As outlined in the title, the main objective of the present workshop No-Cancer 2018 is to discuss the current knowledge in Cancer Cell Biology with the aim of paving the design of more precise and non-invasive methods for early detection of cancer cells and of more effective and personalized therapeutic interventions.

Twelve internationally renowned speakers coming from Belgium, France, Italy, Germany, Spain, Portugal and United States will present their recent research in a two-day scientific marathon. In addition, eight short communications and thirteen flash communications have been selected from the submitted abstracts. The following topics are covered: Oncogenes, onco-suppressors and Non-Coding RNAs in Cancer development; Cancer Cell metabolism and Metabolic cross-talk between cancer cells and stromal cells; Role of diet and of microbiota in cancer progression and response to therapy; Novel non-invasive strategies for early detection of cancer, Genetic alterations predisposing to Cancer; Immune and Drug resistance; cachexia; Clinical trials with novel therapeutics. The invited lectures will focus particularly on ovary cancer, colon cancer, pancreatic cancer, breast cancer, prostate cancer, mesothelioma, and hemato-oncology.

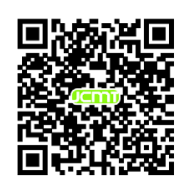


Besides the scientific sessions, participants also can enjoy the social events, which include the city tour, classical music concert and Gala dinner.

Scientific and Social event programs and the Photogallery of the Conference are available at www.isidorolab.com.

This Special Issue of Journal of Cancer Metastasis and Treatment is a collection of the articles and extended abstracts that refer to the lectures presented at the Conference. Commentaries and review articles related to the theme of the Conference are also included.

Ciro Isidoro and Gianluca Gaidano, Guest Editors

\section{Role of canonical and non-canonical signaling in fibroblast to cancer - associated fibroblast transition}

Rangasudhagar Radhakrishnan ${ }^{1}$, Ji Hee Ha ${ }^{1}$, Muralidharan Jayaraman ${ }^{1}$, Katherine M. Moxley ${ }^{1}$, Jinsong Liu ${ }^{2}$, Ciro Isidoro ${ }^{3}$, Yong Sang Song ${ }^{4}$, Danny N. Dhanasekaran ${ }^{1}$

${ }^{1}$ Stephenson Cancer Center, the University of Oklahoma Health Sciences Center, Oklahoma City, OK 73104, USA.

${ }^{2}$ Department of Pathology, the University of Texas M. D. Anderson Cancer Center, Houston, TX 77030, USA.

${ }^{3}$ Università del Piemonte Orientale, Novara 28100, Italy.

${ }^{4}$ Cancer Research Institute, Seoul National University, College of Medicine, Seoul 151-921, South Korea.

Cancer is identified as the second leading cause of death worldwide with an estimated 9.6 million deaths in 2018. The mortality rate of cancer is primarily due to disease recurrence and therapy resistance. Recent studies have shown that the normal stroma in the cancer tissue is converted into an "activated" cancerpromoting niche by the cancer cells facilitating aggressive cancer growth. Normal fibroblasts in the vicinity of the cancer cells are primary recruits in this activation process. "Activated" fibroblasts, defined as cancer associated fibroblasts (CAFs), play a critical role in cancer progression, metastasis, and therapy resistance. Therefore, identifying the mechanisms underlying the conversion of normal cells to CAFs is of critical importance to define novel effective therapeutic targets. Using normal fibroblasts and patient-derived ovarian CAFs, we have identified a role for both lysophosphatidic acid-receptor (LPAR) mediated canonical signaling pathways and lncRNA-hub mediated non-canonical signaling pathways in the induction and maintenance of CAF phenotype in normal fibroblasts. Our studies demonstrate the potential interplay between LPA-LPAR signaling-hub and lncRNA-signaling hub in the maintenance of the CAF-phenotype in ovarian cancer. Our results provide evidence that targeted inhibition of this signaling nexus in CAFs may represent an adjuvant therapy in ovarian cancer.

\section{Obesity-associated alterations of adipose tissue microenvironment and colorectal cancer}

\section{Sandra Gessani, Manuela Del Cornò, Lucia Conti, Gloria Donninelli, Barbara Varano, Beatrice Scazzocchio, Rosaria Varì, Massimo D’Archivio, Roberta Masella}

Istituto Superiore di Sanità - Center for Gender-Specific Medicine, Rome 00161, Italy.

Introduction: Obesity, a low-grade inflammatory condition, is a major risk factor for the development of several pathologies including colorectal cancer (CRC). Adipose tissue (AT) is recognized as a key endocrine 
organ regulating metabolic/immune processes with a central role in obesity-associated morbidities. AT is composed of different cell types, i.e., adipocytes and almost a full spectrum of immune cells whose function is changed in obesity. The AT inflammatory process affects adipocyte metabolism and secretory profile and promotes activation of AT resident immune cells. The obesity-associated changes of this tissue are consistent with an emerging concept that immune and metabolic systems are interconnected. Notably, dietary patterns have been associated with increased/decreased CRC risk highlighting the importance of nutrients in cancer prevention.

Experimental model: Visceral AT samples collected from lean and obese subjects affected or not by CRC were assessed for immune cell, inflammatory and fatty acid (FA) profile as well as secretory function.

Results: Alterations of AT microenvironment including FA profile, inflammatory status, immune cell and secretory pattern are found in obesity and CRC. Dietary polyunsaturated FA endowed with anti- or proinflammatory properties are able to attenuate or exacerbate, respectively, AT inflammation.

Conclusion: AT inflammation has a key role in carcinogenesis and hyper-activated inflammatory pathways in adipocytes can subvert immune surveillance. Dissecting the complexity of events associated with and/ or driving cancer development in obesity will open new avenues for lifestyle-targeted CRC prevention strategies.

\section{Monocarboxylate Transporters as targets for cancer therapy}

\section{Fátima Baltazar ${ }^{1,2}$}

${ }^{1}$ Life and Health Sciences Research Institute (ICVS), School of Medicine, University of Minho, Campus de Gualtar, Braga 4710-057, Portugal.

${ }^{2}$ ICVS/3B's - PT Government Associate Laboratory, Braga/Guimarães 4806-909, Portugal.

Preference for glucose metabolism is a common feature of cancer cells. They rely mainly on glycolysis for adenoside triphosphate (ATP) production, with increased glucose uptake and lactic acid production, leading to acidification of the microenvironment. This metabolic phenotype is associated with features of cancer aggressiveness, including invasion, metastasis, evasion from the immune system, angiogenesis and resistance to therapy. To cope with the high production of acids, cancer cells depend on the activity of proton exchangers and transporters, which export protons to the microenvironment. Among these, are monocarboxylate transporters (MCTs), which play a dual role in tumours, by removing lactate from the cancer cells and also helping in the regulation of intracellular $\mathrm{pH}$. Thus, considering their role in cancer, MCTs represent attractive targets in cancer therapy.

We have studied the expression of MCTs in a variety of human cancer tissues including glioblastoma (GBM) by immunohistochemistry and also blocked MCT activity in different in vitro and in vivo models. MCT1 and MCT4 were found to be overexpressed in human GBM samples compared with diffuse astrocytomas and non-neoplastic samples. MCT1 targeting decreased cell glycolytic metabolism, migration, and invasion, induced cell death and sensitized GBM cells to temozolomide in vitro, and reduced tumour size and angiogenesis in vivo. Additionally, MCT1 is involved in the crosstalk between glioma cells and endothelial cells, and MCT1 targeting either in the tumour cells or endothelial cells decreased endothelial cell proliferation, migration and vessel assembly.

MCT1 is overexpressed in human gliomas and its inhibition decreased cancer cell aggressiveness and sensitized cancer cells to chemotherapy. Additionally, MCT1 mediates endothelial cell metabolic 
reprograming, and, thus, targeting MCT1 in both tumour cells and brain endothelial cells may be a promising therapeutic strategy for the treatment of GBM.

\title{
4. Metformin: toward drugging the metabolic control of epigenetics in cancer
}

\section{Javier A. Menendez}

Catalan Institute of Oncology-Girona Biomedical Research Institute, Girona 17007, Spain.

There is a growing appreciation that metabolic rewiring affects the epigenome in a manner that facilitates cancer formation, progression, and therapeutic resistance. An improved understanding of how the interplay between cell metabolism and the epigenome regulates major cell fate decisions such as cell differentiation, proliferation, and/or cell death, might radically amend the way we prevent and treat cancer. Metformin, a biguanide derivative that has long been a cornerstone in the treatment of type 2 diabetes (T2D), could help to accelerate the development of novel strategies capable of therapeutically tuning the metabolismepigenome axis to battle cancer. This talk will summarize the most recent evidence collected in our laboratory unraveling the capacity of metformin to operate as a poly-therapeutic agent targeting the biologic machinery in charge of the metabolic recoding of cancer epigenetics. On the one hand, metformin can alter the abundance of mitochondrial metabolites that are substrates of chromatin-modifying enzymes (e.g., acetyl-CoA for histone acetyltransferases) by altering the energy status of the cell downstream of its primary inhibitory action on mitochondrial respiratory complex I. On the other hand, biocomputational approaches based on artificial intelligence coupled to experimental validation reveal that metformin is: (1) a direct SIRT1-activating compound that improves the catalytic efficiency of SIRT1-mediated deacetylation in cancer-prone conditions of low $\mathrm{NAD}^{+}$; and (2) a potent regulator of S-adenosyl methionine - mediated methylation reactions via direct and specific inhibition of a central reaction of the folate cycle, namely the conversion of serine to glycine by the mitochondrial serine hydroxymethyltransferase 2 enzyme. The biguanide metformin, which, sixty years after its introduction in Europe as a first-line therapeutic for $\mathrm{T} 2 \mathrm{D}$, may now been seen as an archetypal compound aiming at drugging the metabolism-epigenome axis in cancer.

\section{PIK3C2G loss promotes pancreatic cancer development and metabolic rewiring}

\author{
Miriam Martini, Maria Chiara De Santis, Edoardo Ratto, Luca Gozzelino, Abhishek Uttamrao \\ Derle, Paolo Ettore Porporato, Emilio Hirsch
}

Molecular Biotechnology Center, Dip of Mol Biotechnology and Health Science, Torino 10126, Italy.

Introduction: Pancreatic ductal adenocarcinoma (PDAC) is the most lethal cancer across the world, with incidence equaling mortality. A wealth of studies have identified the PI3K/mTOR axis as an important player in PDACs, impacting on tumor growth and metabolism. In particular, PI3K-C2 $\gamma$, differently from other PI3Ks, is mainly expressed in the pancreatic tissue where it plays a critical role in controlling glucose metabolism.

Experimental model: Mouse model of PDAC (K-RASG12D/Trp53R172H/CrePdx1) was crossed with mouse strain lacking PI3K-C2 $\gamma$ expression. Mice were weekly followed for survival, tumor appearance and growth. Tumor lesions were evaluated by histopathological and immunofluorescence analysis. Functional in vitro and in vivo experiments were performed. 
Results: PI3K-C2 $\gamma$ loss was modeled by deleting $P I K 3 C 2 G$ gene in a mouse model of pancreatic cancer (KPC). We found that its PI3K-C2 $\gamma$ loss was sufficient to initiate and promote pancreatic tumor development, strongly reducing mice survival rate (18 weeks vs. 36 weeks) and driving rapid progression to PDAC. In pancreatic tumors reduced expression of $\mathrm{PI} 3 \mathrm{~K}-\mathrm{C} 2 \gamma$ expression was significantly associated with poor survival and resistance to chemotherapy. We reported that lysosomal localization of PI3K-C2 $\gamma$ is responsible for mTORC1 inhibition under conditions of serum deprivation. In addition, we also observed that PI3K-C2 $\gamma$ loss promotes the metabolic rewiring of PDAC, through the control of several metabolic factors, including glucose and monocarboxylate transporters and glycolytic enzymes (PKM2, HK2 and LDH). Furthermore, the use of rapalogs in KO KPC delay tumor progression.

Conclusion: $P I 3 K-C 2 \gamma$ is a novel tumor suppressor in pancreatic cancer and the metabolic phenotype of PI3K-C2 $\gamma$-deficient tumors can be exploited for specific therapeutic strategies.

\section{Alleviating the Warburg effect: preliminary clinical results in advanced malignancies resistant to chemotherapy}

\section{Laurent Schwartz}

Assistance Publique des hôpitaux de Paris, 3 avenue Victoria, Paris 75004, France.

Chlorine dioxide and Methylene blue are known generators of free radicals. These free radicals are known to enhance the efficacy of the mitochondria.

The goal of this abstract is to describe the first fourteen patients with advanced uncurable cancer treated, with a combination of metabolic treatment (lipoic acid, hydroxycitrate) and chlorine dioxide. All but one patient had failed conventional chemotherapy. Only three patients underwent concomitant conventional chemotherapy. There was no major side effect but nausea and diarrhea. All but one patient responded to treatment. Two more patients were treated with Methylene Blue and metabolic treatment for advanced tumors. One had low dose chemotherapy, the other one, with metastatic pancreatic cancer had no other treatment. Both responded. Rigourous clinical trials are warranted.

\section{Ketogenic diets during radiotherapy against cancer}

\section{Rainer J. Klement}

Department of Radiation Oncology, Leopoldina Hospital Schweinfurt, Robert-Koch-Straße 10, Schweinfurt 97422, Germany.

The altered glucose metabolism of tumor cells is a long-known phenomenon that also contributes to resistance against radiotherapy by securing ATP production important for DNA repair, promoting tumor repopulation and increasing the pool of anti-oxidative molecules for protection against reactive oxygen and nitrogen species (ROS/RNS). In theory therefore, impairing the glucose metabolism of cancer cells is expected to lead to radiosensitization. Deduced from the hypothesis that cancer is a metabolic disease with mitochondrial dysfunction a hallmark of most tumor cells, ketogenic diets - i.e., diets systemically downregulating glycolysis and supporting ketone body and fat oxidation - have been proposed to selectively target tumor cells which in contrast to normal cells would be weakened when glucose and insulin levels are reduced. While many preclinical studies indeed found evidence for a reduction of tumor glycolytic 
metabolism and growth through ketogenic diets, some others found no, or even pro-tumor effects. However, all studies published to date that combined a ketogenic diet with radiotherapy have found a radiosensitizing effect on tumor cells. Mechanisms include a selective increase in ROS and RNS production, ATP reduction and epigenetic impairment of DNA repair through histone deacetylase inhibition by the ketone body $\beta$-hydroxybutyrate, similar to the short chain fatty acid butyrate. The same mechanisms would not apply to normal tissue which is metabolically flexible and even able to initiate an anti-oxidative stress response when switching from glycolysis to mainly mitochondrial respiration fueled by fatty acids and ketone bodies. The hypothesis is therefore that combining a ketogenic diet with radiotherapy could benefit cancer patients in terms of larger tumor control probability for a given radiation dose without affecting or even decreasing the probability of normal tissue toxicity. While this hypothesis has been confirmed in preclinical studies, clinical data are limited thus far by small patient numbers, lack of control groups and lack of systematically collecting hard clinical endpoints such as local tumor control, overall survival and radiotherapy-induced side effects. However, there is some evidence that ketogenic diets could also benefit cancer patients in terms of better maintaining lean body mass which would be relevant especially for frail patient population such as those with head and neck or esophageal cancer. Given the proven safety of ketogenic diets when applied over several weeks in cancer patients, their combination with radiotherapy (as well as other established cancer treatments) deserves further study with a focus on important clinical endpoints.

\title{
8. Blocking the mitochondrial pyruvate carrier to inhibit lactate uptake by cancer cells and induce tumor radiosensitization
}

\section{Cyril Corbet ${ }^{1}$, Estelle Bastien ${ }^{1}$, Lionel Mignion², Bénédicte F. Jordan ${ }^{2}$, Arnaud Marchand ${ }^{3}$, Patrick Chaltin ${ }^{3}$, Holger M. Becker ${ }^{4}$, Olivier Feron ${ }^{1}$}

\author{
${ }^{1}$ Pole of Pharmacology and Therapeutics, Institut de Recherche Expérimentale et Clinique, UCLouvain, Brussels \\ B-1348, Belgium. \\ ${ }^{2}$ Louvain Drug Research Institute, Biomedical Magnetic Resonance Research Group, UCLouvain, Brussels \\ B-1348, Belgium. \\ ${ }^{3}$ CISTIM Leuven, Center for Drug Design and Discovery (CD3) KU Leuven, Heverlee 3001, Belgium. \\ ${ }^{4}$ Division of Zoology/Membrane Transport, FB Biologie, TU Kaiserslautern, D-Kaiserslautern 67653, Germany.
}

Introduction: Lactate-based metabolic symbiosis between glycolytic and oxidative cancer cells is known to facilitate tumor growth. Compounds with the capacity to block this lactate exchange thus represent attractive therapeutic modalities to impact on tumor progression.

Experimental model: Several models, including Xenopus oocytes, 3D tumor spheroids and human tumor xenografts in nude mice, were combined with state-of-the-art metabolomics strategies (Seahorse respirometry, in vitro ${ }^{13} \mathrm{C}$ tracing experiments, and in vivo hyperpolarized ${ }^{13} \mathrm{C}$-pyruvate monitoring) to characterize the mode of action of $7 \mathrm{ACC} 2$, an anticancer compound originally reported to block lactate influx but not efflux.

Results: We identified 7ACC2 as a potent inhibitor of the mitochondrial pyruvate carrier (MPC) activity which consecutively blocks extracellular lactate uptake by promoting intracellular pyruvate accumulation. Importantly, while both 7ACC2 and the MCT1 inhibitor AR-C155858 efficiently inhibited lactate influx, only the former could also block compensatory oxidative glucose metabolism. Moreover, while in 3D tumor spheroids MCT1 inhibition led to cystostatic effects, MPC activity blockade induced cytotoxic effects. This potent growth inhibitory action associated with exacerbated 7ACC2-mediated metabolic alterations (i.e., blockade of lactate- and glucose-fueled TCA cycle) led to a reduction in hypoxia as proven in spheroids via pimonidazole and carbonic anhydrase IX staining and in vivo through electron paramagnetic resonance 
measurements. We showed that this induced tumor reoxygenation could benefit radiotherapy. Indeed, prechallenge of tumor-bearing mice with 7ACC2 considerably improved the anticancer efficacy of either single high dose or fractionated low dose radiation therapy.

Conclusion: This study positions MPC as control point for lactate metabolism and expands on the anticancer potential of MPC inhibition.

\title{
9. The anti-neoplastic effect of triiodothyronine on hepatocellular carcinoma is preceded by
} reversion of the Warburg metabolism and inhibition of the pentose phosphate pathway

\author{
Andrea Perra ${ }^{1}$, Marta Anna Kowalik ${ }^{1}$, Lavinia Cabras ${ }^{1}$, Silvia Giordano ${ }^{2}$, Andrea Rasola ${ }^{3}$, \\ Amedeo Columbano ${ }^{1}$ \\ ${ }^{1}$ Department of Biomedical Sciences, University of Cagliari, Cagliari 09124, Italy. \\ ${ }^{2}$ Department of Biomedical Sciences, University of Padova, Padova 35122, Italy. \\ ${ }^{3}$ Department of Oncology, University of Torino, IRCCS Candiolo, Candiolo 10060, Italy.
}

Introduction: Liver carcinogenesis, from its very early steps, is characterized by reduced activation of thyroid hormone nuclear receptors (THRs), and an altered glucose catabolism that leads to a Warburg metabolism and a strong induction of the pentose phosphate pathway (PPP). The administration of the THRs agonist, triiodothyronine (T3), reverts the preneoplastic hepatocytes to a fully differentiated phenotype and prevents the development of hepatocellular carcinomas (HCC), but its effects on glucose metabolism have not yet been fully explored. Given the strong link between THRs activation and metabolism, the aim of this study is to verify whether T3 is able to restore the physiological PPP activity and energetic metabolism in neoplastic liver.

Experimental model: Rats were subjected to the Resistant-Hepatocyte model of liver carcinogenesis and treated with T3 at 10 weeks, when preneoplastic nodules reached the maximal expansion, or at 10 months after initiation, when HCCs appeared. Rats were killed after 4 or 7 days of T3 treatment.

Results: The treatment with T3 for 7 days resulted in a reduction in the number of preneoplastic lesions. This effect was preceded by a change in the expression of key genes regulators of the glucose metabolism. The immunohistochemical and enzymatic study of liver sections confirmed that the reversion of the Warburg phenotype and inhibition of the PPP occurs before the disappearance of the preneoplastic lesions. The same effect was evident also in fully developed HCCs.

Conclusion: Our results indicate that, at least in part, the antineoplastic effect of T3 depends on the ability of the activated THRs to revert the Warburg phenotype together with an inhibition of the PPP.

\section{The interaction between the transcription factor STAT3 and the $\mathrm{Ca}^{2+}$ channel IP3R3 regulates $\mathrm{Ca}^{2+}$ release from the endoplasmic reticulum and apoptosis}

\author{
Lidia Avalle ${ }^{1, *}$, Annalisa Camporeale ${ }^{1, *}$, Giampaolo Morciano ${ }^{2, *}$, Elena Ghetti ${ }^{1}$, Valeria Orecchia $^{1}$, \\ Carlotta Giorgi ${ }^{2}$, Paolo Pinton ${ }^{2, *}$, Valeria Poli ${ }^{1, *}$ \\ ${ }^{1}$ Department of Molecular Biotechnology and Health Sciences, University of Torino, Torino, 10126, Italy. \\ ${ }^{2}$ Department of Morphology, Surgery and Experimental Medicine, University of Ferrara, Ferrara, 44121, Italy. \\ ${ }^{*}$ Equal contribution.
}


In many tumors the signal transducer and activator of transcription (STAT) 3 has been found to be constitutively active exerting two different functions, both required for cell transformation: (1) activating transcription of its canonical target genes; and (2) regulating energy metabolism and mitochondrial functions. These different activities are mediated by distinct phosphorylation events. In particular, phosphorylation on residue Y705 is responsible for the nuclear activities, while phosphorylated S727 is involved in regulating OXPHOS activities via mithocondrial localization.

We recently reported the localization of STAT3, in both its serine- and tyrosine-phosphorylated forms, to the endoplasmic reticulum (ER) in breast cancer cell lines displaying constitutive activation of this factor. In these cells, S727-phosphorylated STAT3 is able to reduce ER $\mathrm{Ca}^{2+}$ storage and release, thus contributing to resistance to $\mathrm{Ca}^{2+}$ mediated apoptosis upon oxidative stress stimuli. Indeed, $\mathrm{Ca}^{2+}$ release from the ER represents a crucial event in the regulation of life/death decision of cells, and its regulation is mainly exerted by inositol 1,4,5-trisphosphate receptors, and in particular by type 3 (IP3R3), a calcium channel that in response to IP3 stimulation allows $\mathrm{Ca}^{2+}$ transfer from the ER to the mitochondrial associated membranes. We observed that STAT3 can physically interact with IP3R3. Moreover, STAT3 silencing increases IP3R3 expression by decreasing IP3R3 proteasoma-mediated degradation, suggesting that constitutively active STAT3 can inhibit apoptosis by regulating IP3R3 abundance and activity. Accordingly, we found an inverse correlation between IP3R3 and STAT3 protein levels in basal-like breast cancers, a cancer subtype that often shows constitutive STAT3 activation. All together, these data indicate that S727-phosphorylated STAT3 can promote IP3R3 degradation via direct interaction, thus reducing $\mathrm{Ca}^{2+}$ exit from the ER and conferring resistance to apoptosis.

Thus, in addition to the previously characterized nuclear and mitochondrial functions, we described a novel contribution of STAT3 constitutive activation in conferring aggressiveness to breast cancer cells, and in particular to basal-like breast cancers. This is exerted via localization of STAT3 to the ER, interaction with IP3R3 and regulation of its degradation, that leads to decreased calcium release and thus resistance to apoptosis.

\title{
11. Targeting acidic, nutritional and oxidative stresses in cancer
}

\author{
Ibtissam Marchiq ${ }^{1}$, Masa Zdralevic ${ }^{1}$, Milica Vucetic ${ }^{2}$, Scott K. Parks ${ }^{2}$, Jacques Pouysségur ${ }^{1,2}$ \\ ${ }^{1}$ Institute for Research on Cancer and Aging, Nice (IRCAN) University of Nice, Centre A. Lacassagne, 33 \\ avenue de Valombrose, Nice 06189, France. \\ ${ }^{2}$ Medical Biology Department, Centre Scientifique de Monaco (CSM), Monaco 98000, France.
}

In metazoans, sensing the availability of oxygen and key nutrients (glucose, amino acids, fatty acids) is integrated with growth factor and hormone signaling. This multiple nutrient and energy checkpoint converges on the activation of the master protein kinase TORC1, critical for engaging cells in the cell cycle and promoting growth. Cells have evolved sophisticated regulatory systems to rapidly respond to several lethal stressors including metabolic acidosis, nutritional depletion and reactive oxygen species. Cancer cells respond in multiple ways to escape and thrive these microenvironment stresses thus offering several strategies to combat cancer resilience before and after therapeutic treatment.

In this lecture we will discuss how we can exploit cancer vulnerabilities (metabolic tumor acidosis, amino acid depletion and oxidative stress) to propose novel anticancer targets capable to either arrest tumor growth or to kill cancer cells. 


\title{
12. The role of diet related short chain fatty acid acetate in colorectal cancer: therapeutic implications
}

\section{Ana Preto}

Centre of Molecular and Environmental Biology (CBMA), Department of Biology, School of Sciences, University of Minho, Braga 4710-057, Portugal.

Colorectal cancer (CRC) is an important public health concern worldwide, particularly among populations that adopt Western-style diets. The use of dietary propionibacteria found in dairy products, which produce short-chain fatty acids (SCFA), has been suggested as a possible strategy in the prevention and therapy of CRC. The SCFA acetate has been proved by us and others to induce apoptosis in CRC cells. Our group has been focusing on unravelling the mechanisms underlying acetate-induced apoptosis and on understanding the precise role of acetate in CRC cells.

We showed that acetate induces partial lysosome membrane permeabilization with specific cathepsin $\mathrm{D}$ (CatD) release to the cytosol in CRC cells. We verified that CatD has an anti-apoptotic role by the degradation of damaged mitochondria when autophagy is impaired, protecting CRC cells from acetateinduced apoptosis. Moreover, we demonstrated that acetate enters CRC cells by a sodium dependent monocarboxylate transporter 1 and passive diffusion by aquaporins. We also found that MCT-1 and/or MCT-2 seems to mediate acetate transport in CRC cells exposed to acetate. Additionally, we observed that acetate upregulates MCTs expression and promotes plasma membrane re-localization of MCT-1 and triggers changes in glucose metabolism. Further, we explored the combined treatment of acetate with the glycolysis inhibitor $3 \mathrm{BP}$ and we demonstrated that 3BP potentiates acetate-induced apoptosis in CRC cells.

Our results established a protective role of CatD in acetate-induced apoptosis which could negatively impact the efficacy of acetate. Thus, the use of CatD inhibitors in combination with strategies to increase acetate concentrations in the colon, like nutraceuticals, should be explored. Our findings also support a novel approach for CRC therapy based on the association of acetate with $3 \mathrm{BP}$ or other anti-cancer agents whose transport is mediated by MCTs.

\section{Implementing new diet formulations in order to shape microbiota and reverse chemoresistance in the frame of pancreatic cancer}

\author{
Valerio Pazienza, Concetta Panebianco, Kaarel Adamberg, Vilu Raivo, Madis Jaagura, Chiara \\ Saracino, Signe Adamberg, Anna Grazia Di Chio
}

Gastroenterology Unit IRCCS “Casa Sollievo della Sofferenza” Hospital San Giovanni Rotondo, Foggia 71013, Italy.

Introduction: Despite recent advances in treatment options, pancreatic cancer (PC) remain a highly malignant disease and due to its poor prognosis it is ranked as the fourth leading cause of cancer-related deaths worldwide. We have previously demonstrated that manipulating the dietary intake of total amount of calories (using a short-term fasting cycles approach) or replacing carbohydrate with resistant starch have the potential to improve the efficacy of chemotherapy against PC. The aim of this talk is to clarify the effect of the engineered resistant-starch (ERS) mimicking diet on the growth of cancer cell lines in vitro, on the composition of fecal microbiota, and on tumor growth in an in vivo pancreatic cancer mouse xenograft model. Moreover we will shed light on the interaction between the microbiota and chemotherapeutic drugs. 
Experimental model: Pancreatic cancer xenograft mice were subjected to different dietary approaches such as intermittent fasting cycles or ERS diet to assess tumor growth as compared to mice fed with a standard diet. The composition and activity of fecal microbiota and metabolomics profiles were further analyzed by next generation sequencing (NGS) technology and LC-MS analysis of serum respectively.

Results: Pancreatic cancer cells cultured in fasting mimicking medium and ERS diet-mimicking medium impacted the proliferation pathway and the nutrients sensitive pathway decreasing the levels of phosphoERK1/2 and phospho-mTOR. As compared to those cultured in standard medium. Consistently, xenograft pancreatic cancer mice subjected to modifies dietary approaches displayed significant retardation in tumor growth and a significant shift in microbiota profiles towards butyrate producer's microorganisms.

Conclusion: It is important to modulate dietary habits and apply new dietary intervention in order to modulate the composition and metabolism of mouse fecal microbiota which is one of the key organs involved in the chemotherapy response. All these results indicate that engineered dietary interventions may potentially reveal useful in fighting human cancers and could be supportive as a synergistic approach to improve the efficacy of existing drugs in pancreatic cancer therapy.

\title{
14. Effects of selective targeting of mitochondria in experimental cancer and chemotherapy- induced cachexia
}

\author{
Riccardo Ballarò ${ }^{1,2}$, Marc Beltrà ${ }^{1,2}$, Paola Costelli ${ }^{1,2}$, Hazel Szeto ${ }^{3}$, Fabio Penna ${ }^{1,2}$ \\ ${ }^{1}$ Department of Clinical and Biological Sciences, Experimental Medicine and Clinical Pathology Unit, \\ University of Turin, Turin 10126, Italy. \\ ${ }^{2}$ Interuniversity Institute of Myology, Milano 20132, Italy. \\ ${ }^{3}$ Mitochondrial Therapeutics Consulting, New York, NY 10016, USA.
}

Cachexia is a syndrome frequently occurring in cancer patients. The main features are loss of muscle mass and function associated with negative energy balance. In this regard, cachectic tumor-bearing animals show altered mitochondria and reduced oxidative capacity in the muscle. Mitochondrial homeostasis could be further impaired by chemotherapy administration, contributing to muscle wasting.

The present study aimed at evaluating the effects of a mitochondrial-targeted compound (SS-31) on muscle wasting in mice bearing the $\mathrm{C} 26$ tumor in the presence or in the absence of chemotherapy [oxaliplatin + fluoruracil (OXFU)].

Reduced body weight, food intake, muscle mass and function were observed in the untreated tumor hosts. Lifespan in OXFU-treated C26 hosts was almost doubled with respect to untreated animals, however muscle wasting was exacerbated. SS-31 administration was able to counteract body weight loss, anorexia and the reduction of myofiber dimensions, while the protection against muscle mass depletion was only slight. As for markers of mitochondria, both treated and untreated C26 hosts showed that the expression of PGC-1 $\alpha$, cytochrome c, SDH and cardiolipin levels were reduced compared to control animals, and not modified by SS-31. In OXFU-treated tumor-bearing mice, also the SDH total activity and ATP content were reduced. SS31 increased SDH activity and ATP content in untreated C26 hosts. The modulations of the mitochondrial compartment in the C26-bearing mice were also associated with markedly decreased protein synthesis, that improved after administration of SS-31.

In conclusion, these results suggest that targeting mitochondria could be an effective strategy to counteract cancer cachexia, partially preventing the loss of body weight and food intake, improving muscle atrophy, muscle oxidative capacity and energy wasting in mice hosting the C26 tumor in the absence of 
chemotherapy. Further investigation and treatment optimization is required in order to demonstrate a potential effectiveness of SS-31 in the chemotherapy-treated tumor-bearing mice.

\title{
15. L1CAM gene overexpression is associated with platinum-resistance in high-risk endometrial carcinoma
}

\author{
Eliana Bignotti ${ }^{1,2}$, Antonella Ravaggi ${ }^{2,3}$, Martina Ratti ${ }^{1}$, Germana Tognon ${ }^{1}$, Paola Todeschini ${ }^{1,2}$, \\ Laura Zanotti ${ }^{2,3}$, Enrico Sartori ${ }^{3}$, Franco Odicino ${ }^{3}$, Chiara Romani ${ }^{4}$ \\ ${ }^{1}$ U.O. Obstetrics and Gynecology, ASST Spedali Civili di Brescia, Brescia 25123, Italy. \\ ${ }^{2} I M M$ "A. Nocivelli", ASST Spedali Civili di Brescia, Brescia 25123, Italy. \\ ${ }^{3}$ Division of Obstetrics and Gynecology, University of Brescia, Brescia 25121, Italy. \\ ${ }^{4}$ Molecular and Translational Medicine Department, University of Brescia, Brescia 25121, Italy.
}

Introduction: L1 cell adhesion molecule (L1CAM) expression has been reported associated with high-grade disease and non-endometrioid histology, as well as poor prognosis, in endometrial carcinoma (EC). These high-risk EC types have frequently already spread outside the uterus when diagnosed and, after an extensive surgery, are often treated with chemo and radiation therapy. We hypothesized that L1CAM gene expression could discriminate, among poor outcome EC patients, those who do and who do not respond to adjuvant platinum-based chemotherapy.

Experimental model: Using an efficient multiplex qRT-PCR, we test L1CAM mRNA expression on 117 EC and 16 normal endometrial (NE) flash-frozen tissues, with HPRT1 and PPIA as reference genes.

Results: L1CAM mRNA was significantly overexpressed in EC compared to NE tissues $(P=0.02)$, significantly upregulated in G3 vs. G1-2 ECs $(P<0.001)$ and in non-endometrioid vs. endometrioid ECs $(P<0.001)$. Our analysis showed no difference in L1CAM expression of stage I-II $v s$. stage III-IV ECs $(P$ $=0.5$ ). Of the initial $117 \mathrm{EC}$ patients, 47 received chemotherapy on adjuvant setting and were classified as platinum-sensitive and platinum-resistant patients, based on PFI $>12$ months and $<6$ months, respectively. L1CAM gene was significantly overexpressed in resistant vs sensitive EC $(P=0.001)$. Moreover, by means of a multivariate logistic regression model, we found L1CAM gene overexpression as an independent indicator of the probability to harbor a platinum-resistant EC $(P=0.047, \mathrm{OR}=3.5)$. In addition, univariate and multivariate survival analysis showed L1CAM gene upregulation associated with poor outcome, in terms of progression-free survival and disease-specific survival.

Conclusion: Our results suggest L1CAM gene expression as a potential prognostic marker and a predictive biomarker of platinum-response in high-risk EC patients.

\section{Resveratrol counteracts ovarian cancer cell migration stimulated by interleukin- 6 by limiting glucose uptake}

\section{Chiara Vidoni ${ }^{1}$, Alessandra Ferraresi ${ }^{1}$, Letizia Vallino ${ }^{1}$, Andrea Esposito ${ }^{1}$, Eleonora Secomandi ${ }^{1}$, Danny N. Dhanasekaran ${ }^{2}$, Ciro Isidoro'}

${ }^{1}$ Laboratory of Molecular Pathology and Nanobioimaging, Department of Health Sciences, Università del Piemonte Orientale "A. Avogadro", Via Solaroli 17, Novara 28100, Italy.

${ }^{2}$ Stephenson Cancer Center and Department of Cell Biology, The University of Oklahoma Health Sciences Center, Oklahoma City, OK 73104, USA. 
Introduction: IL-6, a pro-inflammatory cytokine produced by cancer-associated fibroblasts, increases the proliferative and invasive properties of ovarian cancer cells. Glucose metabolism is altered in ovarian cancer cells and permits fast proliferation and survival. Resveratrol (RV) is a naturally occurring polyphenol with the potential to inhibit cancer cell migration.

Results: Here, we found that IL-6 enhances ovarian cancer cell migration, while RV and deprivation of glucose reduce cell motility. In particular, IL-6 stimulates glucose uptake along with cell migration, while RV abrogates this effect through the reduction of GLUT1 plasma membrane translocation and glucose internalization. Further, the cells exposed to IL- 6 at the migration front show an increased expression of $\mathrm{N}$-cadherin over E-cadherin, and this effect is reverted by RV exposure. Accordingly, the expression of TWIST1, a regulator of epithelial-to-mesenchymal transition (EMT), is reduced by RV and deprivation of glucose. Transcriptomic and microRNomic analyses revealed that RV up-regulates a subset of miRNAs that have several glucose metabolism regulators as targets. In particular, we found that RV abrogates the transcription of ZEB1, Hexokinase 2 and FOXM1.

Conclusion: Our data indicate that RV counteracts glucose metabolism negatively impinging on ovarian cancer cell migration induced by IL- 6 .

\title{
17. FXYD5 is a predictor of short-term survival in high-grade serous ovarian carcinoma
}

\section{Antonella Ravaggi ${ }^{1,2, *}$, Renata A Tassi ${ }^{1, *}$, Angela Gambino ${ }^{2}$, Laura Ardighieri ${ }^{3}$, Mattia Bugatti ${ }^{3}$, Chiara Romani ${ }^{4}$, Paola Todeschini ${ }^{1}$, Laura Zanotti ${ }^{1}$, Francesco Gebbia ${ }^{2}$, Elisa Picardo ${ }^{5}$, Dionyssios Katsaros ${ }^{5}$, Eliana Bignotti ${ }^{1,6}$, Chiara Romualdi ${ }^{7}$, Enrico Sartori ${ }^{2}$, Franco Odicino ${ }^{2}$}

\author{
"A. Nocivelli" Institute of Molecular Medicine, ASST Spedali Civili of Brescia, Brescia 25123, Italy. \\ ${ }^{2}$ Division of Obstetrics and Gynecology, University of Brescia, Brescia 25121, Italy. \\ ${ }^{3}$ Department of Pathology, ASST Spedali Civili of Brescia, Brescia 25123, Italy. \\ ${ }^{4}$ Department of Molecular and Translational Medicine, University of Brescia, Brescia 25121, Italy. \\ ${ }^{5}$ Department of Surgical Sciences, Gynecologic Oncology, Città della Salute and S Anna Hospital, University of \\ Turin, Turin 10126, Italy. \\ ${ }^{6}$ Division of Obstetrics and Gynecology, ASST Spedali Civili of Brescia, Brescia 25123, Italy. \\ ${ }^{7}$ Department of Biology, University of Padova, Padova 35122, Italy. \\ ${ }^{\star}$ Equal contribution.
}

Introduction: High-grade serous ovarian carcinoma (HGSOC) is generally associated with a very dismal prognosis. Nevertheless, patients with similar clinicopathological characteristics can have markedly different clinical outcomes. We aim to identify the molecular determinants influencing survival by comparing the gene expression patterns of two patient cohorts characterized by extreme overall survival.

Experimental model: We determined the gene expression profiles of 12 HGSOC long-term and 27 shortterm survivors (training set) by microarray chips. By a generalized linear model with cross-validation, we generated a prognostic gene signature that was further evaluated on the entire "The Cancer Genome Atlas" (TCGA) ovarian cancer dataset. The resulting genes were then verified on an independent cohort of 29 HGSOC flash frozen samples (validation set) by RT-qPCR, and in a panel of 38 formalin fixed paraffin embedded HGSOC tissues by immunohistochemistry (IHC).

Results: We identified a ten-gene prognostic signature able to correctly assign $98 \%$ of patients of the training set within their survival class. By "in silico" validation on TCGA microarray dataset, we confirmed the overexpression in short term survivors of FXYD domain containing ion transport regulator 5 (FXYD5), 
one of the 10 top score genes of the signature. The prognostic power of FXYD5 was also successfully validated, both at mRNA and protein level by RT-qPCR and IHC, respectively on the training and validation sets. Moreover, FXYD5 overexpression was significantly associated with platinum resistance and cancer progression.

Conclusion: We demonstrated the consistent overexpression of FXYD5 in HGSOC short-term survivors compared to long-term ones. FXYD5 may become a useful predictive marker for a more accurate selection of HGSOC patients for adjuvant treatments, and a possible target for antibody-drug conjugated anticancer agents, as recently demonstrated for thyroid cancer cell lines.

\title{
18. Claudin-7 downregulation is predictive of distant metastases in high-grade serous ovarian carcinoma patients
}

\section{Chiara Romani ${ }^{1}$, Valentina Zizioli ${ }^{2}$, Marco Silvestri ${ }^{3}$, Michela Corsini ${ }^{1}$, Laura Ardighieri ${ }^{4}$, Paola Todeschini ${ }^{5}$, Sergio Marchini ${ }^{6}$, Maurizio D'Incalci ${ }^{6}$, Laura Zanotti ${ }^{5}$, Antonella Ravaggi ${ }^{5}$, Franco Odicino $^{2}$, Enrico Sartori ${ }^{2}$, Alessandro Davide Santin ${ }^{7}$, Stefania Mitola ${ }^{1}$, Eliana Bignotti ${ }^{2, *}$, Stefano Calza $^{8, *}$}

\author{
${ }^{1}$ Department of Molecular and Translational Medicine, University of Brescia, Brescia 25121, Italy. \\ ${ }^{2}$ Division of Gynecologic Oncology, ASST Spedali Civili, Brescia 25123, Italy. \\ ${ }^{3}$ Fondazione IRCCS Istituto Nazionale dei Tumori di Milano, Department of Applied Research and \\ Technological Development, Biomarkers Unit, Milan 20133, Italy. \\ ${ }^{4}$ Department of Pathology, ASST Spedali Civili di Brescia, Brescia 25123, Italy. \\ "Angelo Nocivelli" Institute of Molecular Medicine, Division of Gynecologic Oncology, University of Brescia, \\ Brescia 25121, Italy. \\ ${ }^{6}$ Department of Oncology, IRCCS, "Mario Negri” Institute for Pharmacological Research, Milan 20156, Italy. \\ ${ }^{7}$ Department of Obstetrics, Gynecology and Reproductive Sciences, Yale University School of Medicine, 333 \\ Cedar Street, PO Box 208063, New Haven, CT 06520-8063, USA. \\ ${ }^{8}$ Department of Molecular and Translational Medicine, Unit of Biostatistics and Bioinformatics, University of \\ Brescia, Brescia 25121, Italy. \\ ${ }^{\star}$ Equal contribution.
}

Introduction: High-grade serous carcinoma (HGSOC) is the most frequent and lethal ovarian carcinoma histotype. Although the abdominal dissemination is considered the most common, distant metastases occur in about $30 \%$ of patients with newly diagnosed or recurrent HGSOC. No tumor marker can currently predict the risk of distant metastasis in HGSOC. Tight-junction protein claudin-3, -4 and -7 are frequently dysregulated in HGSOC and functionally related to cancer progression to a metastatic disease. Here we analyze claudin- $-3,-4$ and -7 expression as markers of distant metastasis.

Experimental model: Claudin expression was evaluated in 105 primary HGSOC tissues, 14 normal ovarian and 26 normal fallopian tube epithelia by quantitative RT-PCR and immunohistochemistry, and correlated with clincopathological features. Gene set enrichment analysis was performed on microarray-generated gene expression data to investigate key pathways in patients with distant metastasis.

Results: Claudin-3, -4 and -7 expression levels are decreased in HGSOC compared to normal tubal epithelium, currently considered alternative source of such tumors. Decreased expression of claudin-7 is seen in tumors from women who develop distant recurrence $(P=0.016)$, mainly by hematogenous route $(P$ $=0.025)$. The estimated reduction in the probability of distant disease is of $39 \%$ per unit increase in the level 
of claudin-7 (AUC 0.659, $P=0.03$ ). Genes involved in hypoxia and angiogenesis processes result strongly associated to hematogenous recurrence $(P=0.012)$.

Conclusion: Claudin-7 decreased expression in primary tumor tissues is a significant predictor of distant metastasis in HGSOC patients.

\title{
19. Glutamine synthetase-negative multiple myeloma cells secrete glutamate and shape the bone marrow niche
}

\author{
Martina Chiu, Denise Toscani, Giuseppe Taurino, Fabrizio Accardi, Nicola Giuliani, Ovidio \\ Bussolati
}

Department of Medicine and Surgery (DiMeC), University of Parma, Parma 43121, Italy.

\begin{abstract}
Altered metabolism, a hallmark of cancer, also impacts on non-cancer cells of tumor microenvironment, driving them towards a pro-tumor behavior. In multiple myeloma (MM) patients, bone marrow glutamine (Gln) is lowered, while glutamate (Glu) and ammonium increase. In most MM patients, Glutamine Synthetase (GS), the enzyme that catalyzes Gln synthesis from Glu and ammonium, is lowered in neoplastic plasma cells. GS is also down-regulated during normal osteoblast differentiation from mesenchymal stromal cells (MSC). Since bone lesions of MM patients are characterized by impaired osteogenesis, we hypothesize that MM cells negatively affect osteoblasts through the peculiar low-Gln, high-Glu bone marrow microenvironment and the manipulation of GS expression in niche cells.
\end{abstract}

Human MM cell lines (HMCLs) and immortalized MSC were grown in Glu-free $\alpha$ MEM and used in monoor co-cultures. Gene expression was assessed at either mRNA and protein level. Amino acid levels were determined with a colorimetric test (extracellular) and LC-MS/MS (intracellular).

Analysis of a dataset of 323-sample plasma cell dyscrasias indicated that GS expression is downregulated during MM progression, with less than 20\% MM patients with GS-positive CD138 ${ }^{+}$cells. Also most HMCLs, but not MM1.S and U266, have a negligible GS expression. While both low- and high-GS HMCLs exhibited a high consumption of Gln and a comparable glutaminolysis, low-GS HMCLs exploited roughly 50\% of Gln to secrete Glu. Consistently, the activity of the Glu exchanger xCT was higher in low-GS MM cells. GS is strongly induced in MSC when co-cultured with MM cells.

These preliminary results are consistent with the hypothesis that low GS expression, Gln addiction and Glu secretion of MM cells are functional to osteoblastic differentiation impairment in the BM niche.

\section{Evaluation of the interaction among HPV16 E6 and E7 oncoproteins and the DNA damage sensor 53BP1 in 2D and 3D epithelial cultures by the proxymity ligation assay}

\section{Barbara Azzimonti, Diletta Francesca Squarzanti, Rita Sorrentino, Manuela Miriam Landini, Andrea Chiesa.}

University of Piemonte Orientale (UPO), School of Medicine, Department of Health Sciences, Via Solaroli 17, Novara 28100, Italy.

Introduction: Human papillomaviruses (HPV) group several viruses are able to infect squamous stratified epithelia and cause benign papillomas, warts and anogenital lesions. They also correlate to oropharyngeal 
and anogenital malignancies, mainly promoted by the high risk (HR) $\alpha$-HPV16 E6E7 oncoproteins. Despite scientific progresses and the development of vaccines, these tumors are still common and represent one of the major causes of women's death. Host's cell replication fidelity depends by the DNA damage response (DDR). Unlike from other DNA viruses, HR-HPVs encourage cells proliferation without inactivating the DDR: the mechanisms at the basis haven't been clarified yet. During HPV16 infection, E6 binds and degrades p53 through the E6AP LXXLL domain. Similarly, E7 competes with E2F1-pRb interaction, thus inactivating $\mathrm{pRb}$, and promoting the linking the $\mathrm{pRb}$-like proteins $\mathrm{CBP} / \mathrm{p} 300$ and $\mathrm{p} 107$, that also harbor a LXXLL sequence. Unfortunately, E6 E7 role in the DDR activation is not elucidated yet.

Experimental model: To gain new information, we reproduced an in vitro 3D HPV16-E6E7 infected epithelium, already characterized for HPVs studies, and checked for cellular and viral markers, among them HPV16E6E7 oncoproteins and the double strand breaks sensor 53BP1; we then made a Co-IF for E6 and E7 with 53BP1. Since E6 and E7 both interact with LXXLL containing proteins, we analyzed 53BP1 BRCT2 domain and we explored the binding hypothesis via the in situ PLA technique in 2D in CaSki and E6E7HPV16 keratinocytes and in the 3D model.

Results: The in vitro infected epithelium resembles the in vivo tissue. E6E7HPV16, both in basal and differentiated strata, induce a 53BP1 increase in nuclear foci. After highlighting E6 and E7 co-expression with 53BP1 and a LKVLL sequence within the 53BP1 BRCT2 domain, we demonstrated the binding in all the employed cellular models.

Conclusion: Our results add new information on HPV16 oncoproteins capability in overcoming cellular defense strategies.

\section{Iron metabolism regulates cancer related skeletal muscle wasting}

\section{Erica Mina, Elisabeth Wyart, Myriam Hsu, Paolo Ettore Porporato}

Molecular Biotechnology Centre, Department of Molecular Biotechnology and health sciences, University of Torino, Torino 10126, Italy.

Introduction: Cancer associated cachexia is a syndrome characterized by a significant weight loss, due to metabolic changes affecting skeletal muscle and adipose tissue2018. Given the importance of iron in controlling energy metabolism, we speculated that decreased iron availability occurring in cancer might contribute to skeletal muscle atrophy.

Experimental model: In vitro experiments are performed with $\mathrm{C} 2 \mathrm{C} 12$ myotubes, while in vivo experiments are conducted using Colon-26 carcinoma bearing BALB-C mice.

Results: Interestingly, using Colon-26 carcinoma bearing mice, we found strong alterations in protein and gene expression of iron homeostasis key players in skeletal muscle, notably a downregulation of transferrin receptor 1 (TFR1, the main importer of iron) and an increase of the iron exporter Ferroportin. Coherently, we observed a decreased iron content in both skeletal muscle and spleen while serum iron is increased, suggesting a global mobilization of iron. To further confirm our hypothesis, we created iron-deprived models in vitro using several iron chelators (Desferoxamine, BPS) or by TFR1 knockdown with siRNA and, in line with our in vivo observations, iron depletion in $\mathrm{C} 2 \mathrm{C} 12$ myotubes directly promoted atrophy. Finally, we were able to prevent myotube atrophy by restoring iron transport.

Conclusion: Taken together, these results highlight a previously unknown role for altered iron homeostasis in cancer-induced muscle wasting and provide a potential new therapeutic target. 


\section{Liquid biopsy applications in lymphomas}

Fary Diop ${ }^{1}$, Riccardo Moia ${ }^{1}$, Chiara Favini ${ }^{1}$, Clara Deambrogi ${ }^{1}$, Ahad A. Kodipad ${ }^{1}$, Sruthi Sagiraju', Ramesh Adhinaveni ${ }^{1}$, Abdurraouf M. Mahmoud ${ }^{1}$, Syed Hasan Mosavi ${ }^{1}$, Sreekar Kogila ${ }^{1}$, Simone Favini ${ }^{1}$, Denise Peroni ${ }^{1}$, Marta Castagno ${ }^{1}$, Silvia Rasi ${ }^{1}$, Valeria Spina ${ }^{2}$, Andrea Patriarca ${ }^{1}$, Gloria Margiotta Casaluci ${ }^{1}$, Luca Nassi ${ }^{1}$, Davide Rossi ${ }^{2}$, Gianluca Gaidano ${ }^{1}$

${ }^{1}$ Division of Hematology, Department of Translational Medicine, Amedeo Avogadro University of Eastern Piedmont, Novara 28100, Italy.

${ }^{2}$ Oncology Institute of Southern Switzerland, and Laboratory of Experimental Hematology, Institute of Oncology Research, Bellinzona 6500, Switzerland.

Liquid biopsy is an emerging tool across many types of cancers. This technique consists in the analysis of biomarkers released by tumor cells in the peripheral blood. In lymphomas, the most studied biomarker is circulating tumor DNA (ctDNA), shed into the bloodstream (e.g., plasma) by tumor cells undergoing apoptosis.

Analysis of plasma ctDNA analysis allows serial monitoring of the disease genotype over time and the assessment of the entire tumor heterogeneity at different anatomic sites. Consistently, ultra-deep targeted next generation sequencing of ctDNA from diffuse large B-cell lymphoma (DLBCL) patients correctly identified DLBCL-associated mutations. Moreover, plasma ctDNA genotyping also allows for the recovery of mutations that are undetectable in the tissue biopsy, conceivably because, due to spatial tumor heterogeneity, they are restricted to clones that are anatomically distant from the biopsy site. In Hodgkin lymphoma (HL), the rarity of neoplastic cells in the biopsy has so far limited genomic studies. By using a highly sensitive and robust deep next-generation sequencing approach for ctDNA, the current knowledge of HL has been refined. In addition, similarly to DLBCL, also in HL a fraction of mutations has been detected only in ctDNA but not in the tissue biopsy.

Regarding disease monitoring during the course of treatment, CT/PET scans do not capture all patients designated to relapse. This gap may be filled by ctDNA analysis providing higher sensitivity and ease of sample collection in a radiation free manner. In DLBCL, the 2.5-log drop of ctDNA concentration after two cycles of treatment is an independent predictor of response. Similarly, in HL the 2-log drop of ctDNA after two courses of chemotherapy associated with complete response and complemented the results obtained by the CT/PET scan. Incorporation of both CT/PET and ctDNA monitoring into clinical trials may guide future personalized risk-directed approaches of treatment.

\section{BIRC3 mutations stratify a poor prognostic subgroup in Fludarabine-Cyclophosphamide-}

\section{Rituximab treated chronic lymphocytic leukemia}

Riccardo Moia ${ }^{1}$, Fary Diop ${ }^{1}$, Chiara Favini ${ }^{1}$, Elisa Spaccarotella ${ }^{1}$, Lorenzo De Paoli ${ }^{1}$, Alessio Bruscaggin $^{2}$, Valeria Spina ${ }^{2}$, Lodovico Terzi-di-Bergamo ${ }^{2}$, Francesca Arruga ${ }^{3}$, Chiara Tarantelli ${ }^{4}$, Clara Deambrogi ${ }^{1}$, Simone Favini ${ }^{1}$, Ahad A. Kodipad ${ }^{1}$, Sruthi Sagiraju ${ }^{1}$, Denise Peroni $^{1}$, Francesca R. Mauro ${ }^{5}$, Ilaria Del Giudice ${ }^{5}$, Francesco Forconi ${ }^{6,7}$, Agostino Cortelezzi ${ }^{8}$, Francesco Zaja ${ }^{9}$, Carlo Visco ${ }^{10}$, Annalisa Chiarenza ${ }^{11}$, Gian Matteo Rigolin ${ }^{12}$, Roberto Marasca ${ }^{13}$, Marta Coscia ${ }^{14}$, Omar Perbellini ${ }^{15}$, Alessandra Tedeschi ${ }^{16}$, Luca Laurenti ${ }^{17}$, Marina Motta ${ }^{18}$, Francesco Bertoni ${ }^{4}$, Giovanni Del Poeta ${ }^{19}$, Antonio Cuneo ${ }^{12}$, Valter Gattei ${ }^{20}$, Silvia Deaglio ${ }^{3}$, Mark Catherwood $^{21}$, Robin Foà ${ }^{5}$, Gianluca Gaidano ${ }^{1, *}$, Davide Rossi ${ }^{2, *}$ 
${ }^{1}$ Division of Hematology, Department of Translational Medicine, Amedeo Avogadro University of Eastern Piedmont, Novara 28100, Italy.

${ }^{2}$ Institute of Oncology Research and Oncology Institute of Southern Switzerland, Bellinzona 6500, Switzerland. ${ }^{3}$ Department of Medical Sciences, University of Turin, Turin 10126, Italy.

${ }^{4}$ Università della Svizzera Italiana (USI), Institute of Oncology Research (IOR), Bellinzona 6500, Switzerland.

${ }^{5}$ Hematology, Department of Cellular Biotechnologies and Hematology, Sapienza University, Rome 00185, Italy.

${ }^{6}$ Cancer Sciences Unit, Southampton Cancer Research UK and National Institute for Health Research Experimental Cancer Medicine Centre, University of Southampton, Southampton SO17 1BJ, United Kingdom.

${ }^{7}$ Division of Hematology, University of Siena, Siena 53100, Italy.

${ }^{8}$ Department of Hematology Oncology, Foundation IRCCS Ca' Granda Ospedale Maggiore Policlinico and University of Milan, Milan 20122, Italy.

${ }^{9}$ Clinica Ematologica, DAME, University of Udine, Udine 33100, Italy.

${ }^{10}$ Department of Cell Therapy and Hematology, Ospedale San Bortolo, Vicenza 36100, Italy.

${ }^{11}$ Division of Hematology, Azienda Ospedaliera Universitaria Policlinico-OVE, Catania 95123, Italy.

${ }^{12}$ Hematology Section, Azienda Ospedaliero Universitaria Arcispedale S. Anna, University of Ferrara, Ferrara 44124, Italy.

${ }^{13}$ Division of Hematology, Department of Oncology and Hematology, University of Modena and Reggio Emilia, Modena 41121, Italy.

${ }^{14}$ Division of Hematology, Azienda Ospedaliero Universitaria Città della Salute e della Scienza and University of Turin, Turin 10126, Italy.

${ }^{15}$ Section of Hematology, Department of Medicine, University of Verona, Verona 37129, Italy.

${ }^{16}$ Department of Oncology/Haematology, Niguarda Cancer Center, Niguarda Ca Granda Hospital, Milan 20162, Italy.

${ }^{17}$ Institute of Hematology, Catholic University of the Sacred Heart, Rome 00168, Italy.

${ }^{18}$ Department of Hematology, Spedali Civili, Brescia 25123, Italy.

${ }^{19}$ Department of Hematology, Tor Vergata University, Rome 00133, Italy.

${ }^{20}$ Clinical and Experimental Onco-Hematology Unit, Centro di Riferimento Oncologico, Istituto di Ricovero e Cura a Carattere Scientifico (IRCCS), Aviano 33081, Italy.

${ }^{21}$ Department of Hemato-Oncology, Belfast City Hospital, Belfast BT9 7AB, UK.

${ }^{\star}$ Equal contribution.

Introduction: The current shift of therapy of chronic lymphocytic leukemia (CLL) towards novel targeted agents mandates the identification of new molecular predictors. The aim of this study is to identify new molecular predictors in FCR treated patients and to assess the biological features underlying chemorefractoriness to FCR.

Experimental model: A retrospective multicenter cohort of 287 untreated CLL receiving first-line therapy with FCR was subjected to a targeted NGS approach in 24 most recurrently mutated genes in CLL. The entire non-canonical NF- $\mathrm{KB}$ pathway was assessed by Western blot and by real-time PCR.

Results: $\mathrm{SF}_{3} \mathrm{~B}_{1}$ and $\mathrm{NOTCH} 1$ were the most frequently mutated genes identified in $13.9 \%$ and in $13.6 \%$ of patients respectively, followed by TP53 in 9.4\% and ATM in 6.9\%. BIRC3 was mutated in $3.1 \%$ of patients. By univariate analysis adjusted for multiple comparisons, only BIRC3 mutations (median PFS of 2.2 years; $P<$ 0.001 ) and TP53 mutations (median PFS of 2.6 years; $P<0.0001$ ) identified patients who failed early FCR. By multivariate analysis, BIRC3 mutations maintained an independent risk of progression, with a HR of 2.8 (95\%CI 1.4-5.6, $P=0.004)$. In addition, in vitro studies showed that fludarabine-induced apoptosis in BIRC 3 mutated cells was comparable to samples harboring TP53 mutations. Western blotting analysis of the noncanonical NF- $\kappa \mathrm{B}$ pathway showed that BIRC3 mutated cells was significantly enriched of non-canonical NF$\kappa \mathrm{B}$ target gene and addicted of MAP3K14 overexpression. 
Conclusion: BIRC3 mutations identify a very poor prognostic subgroup of patients that fails FCR similar to patients harboring TP53 abnormalities. If validated, BIRC3 might be used as a new molecular predictor to select high-risk patients for novel frontline therapeutic approaches. From the biological point of view, BIRC3 mutations enhance the non-canonical NF- $\mathrm{B}$ signaling pathway promoting survival, proliferation and chemo-refractoriness.

\title{
24. Peptide nucleic acid-based targeting of microRNAs: possible therapeutic and diagnostic applications for glioblastoma
}

\section{Roberto Gambari}

Department of Life Sciences and Biotechnology, Ferrara University, Ferrara 44124, Italy.

Introduction: MicroRNAs (miRNAs) are small noncoding RNAs regulating gene expression by sequenceselective targeting of mRNAs, leading to translational repression or mRNA degradation. Low miRNA expression is associated with accumulation of target mRNAs; high miRNA content causes low expression of target mRNAs. In cancer, microRNAs are associated with tumor onset and progression.

Experimental model: Targeting oncomiRNAs and metastamiRNAs with biomolecules interfering with their biological activity is of interest and peptide-nucleic acids (PNAs) might be useful. PNAs are DNA analogues in which the sugar-phosphate backbone has been replaced by $\mathrm{N}$-(2-aminoethyl) glycine units, hybridize to RNA with high efficiency, are resistant to proteinases and nucleases, and have been proposed as excellent tools for alteration of gene expression. We have developed novel delivery strategies for PNAs targeting miRNAs, based on the use of PNAs linked to a poly-arginine R8 peptide tail for efficient cellular delivery. As far as cancerrelated model systems, we focused on PNAs targeting miR-221 and miR-222 in glioblastoma cells.

Results: In a first study, a combined treatment of U251, U373 and T98G glioma cell lines was performed with different anti-miRNA PNAs (against miR-221, miR-222 and miR-155). Increased pro-apoptotic effects were obtained with the co-administration of both anti-miR-221 and anti-miR-222 PNAs, or anti-miR-221 and anti-miR-155 PNAs. In a second study, we demonstrated synergistic effects of co-administration of corilagin and a PNA targeting miR-221. In a third approach we performed a combined treatment of glioma U251 cells with the pro-apoptotic pre-miR-124 and the PNA targeting miR-221, showing induction of apoptosis at very high levels.

Conclusion: PNAs might be a relevant therapeutic tool for anti-cancer miRNA-therapy based on inhibition of oncomiRNA and metastamiRNAs, as well as mimicking tumor-suppressor miRNAs (funded by AIRC IG13575 and Horizon 2020 Project ULTRAPLACAD).

\section{Liquid biopsy-based colorectal cancer diagnosis: analysis of a limited panel of miRNA in mice bearing colorectal carcinoma tumor xenografts and in Human plasma samples}

\author{
Jessica Gasparello ${ }^{1}$, Matteo Allegretti ${ }^{2}$, Elisa Tremante ${ }^{2}$, Chiara Papi ${ }^{1}$, Enrica Fabbri ${ }^{1}$, Carla \\ Azzurra Amoreo ${ }^{3}$, Paolo Romania ${ }^{2}$, Elisa Melucci ${ }^{3}$, Katia Messana ${ }^{2}$, Monica Borgatti ${ }^{1}$, Patrizio \\ Giacomini ${ }^{2}$, Roberto Gambari ${ }^{1}$, Alessia Finotti ${ }^{1}$ \\ ${ }^{1}$ Department of Life Sciences and Biotechnology, University of Ferrara, Ferrara 44124, Italy. \\ ${ }^{2}$ Oncogenomics and Epigenetics Unit, Regina Elena National Cancer Institute, Rome 00144, Italy.
}


${ }^{3}$ Pathology, IRCSS Regina Elena National Cancer Institute, Rome 00144, Italy.

Introduction: Due to their high stability in body fluids, circulating tumor microRNAs are proposed as promising biomarkers useful for early tumor diagnosis, prognosis, monitoring, and to predict therapeutic response, in non-invasive liquid biopsy. We investigated the release of miR-141-3p, miR-221-3p and miR-2223p previously associated to colorectal cancer (CRC).

Experimental model: We employed droplet digital PCR to quantify the amount of miRNAs released in: (1) supernatants of three human CRC cell lines (HT-29, LoVo and Ls174T); (2) in plasma of nude mice inoculated with the same three cell lines, in order to obtain tumor xenograft models; (3) in plasma isolated from a heterogeneous group of CRC patients.

Results: MicroRNAs miR-221-3p and miR-222-3p (but not miR-141) in cellular supernatants were proportional to the cellular levels. Interestingly, all three miRNAs are released in plasma of xenografted mice. Using plasma samples from CRC patients, we found that only in $57 \%$ of the cases it was possible to identify a differential expression of at least one of the miRNAs with respect to control subjects.

Conclusion: Our data demonstrate that, despite the three selected miRNAs are not only present in CRC cells and tissues but are also released in extracellular environments, they are not informative for a high proportion of CRC patients. NGS allowed to expand the number of miRNAs differentially expressed in CRC samples. A novel set, constituted of 12 miRNA was demonstrated to be of diagnosis relevance in $94 \%$ of CRC patients samples (funded by AIRC IG13575 and Horizon 2020 Project ULTRAPLACAD).

\title{
26. Sensitivity to asbestos is increased in patients with mesothelioma and pathogenic germline variants in BAP1 or other DNA repair genes
}

\author{
Marika Sculco ${ }^{1}$, Marta Betti ${ }^{1 *}$, Anna Aspesi ${ }^{{ }^{*}}$, Daniela Ferrante ${ }^{2}$, Luisella Righi ${ }^{3}$, Dario Mirabelli ${ }^{4}$, \\ Francesca Napoli ${ }^{3}$, Milena Rondón-Lagos ${ }^{5}$, Elisabetta Casalone ${ }^{6,7}$, Francesca Vignolo Lutati ${ }^{8}$, \\ Paola Ogliara ${ }^{8}$, Paolo Bironzo ${ }^{9}$, Laura Cristina Gironi ${ }^{1}$, Paola Savoia ${ }^{1}$, Antonella Maffè ${ }^{10}$, Silvana \\ Ungari $^{10}$, Federica Grosso ${ }^{11}$, Roberta Libener ${ }^{12}$, Renzo Boldorini ${ }^{13}$, Michele Valiante ${ }^{14}$, Barbara \\ Pasini $^{8}$, Giuseppe Matullo ${ }^{6,7,8}$, Giorgio Scagliotti ${ }^{9}$, Corrado Magnani ${ }^{2}$, Irma Dianzani ${ }^{1}$ \\ ${ }^{1}$ Department of Health Sciences, University of Piemonte Orientale, Novara 28100, Italy. \\ ${ }^{2}$ Unit of Cancer Epidemiology, CPO-Piemonte, Department of Translational Medicine, University of Piemonte \\ Orientale, Novara 28100, Italy. \\ ${ }^{3}$ Department of Oncology, University of Turin at San Luigi Hospital, Orbassano 10043, Italy. \\ ${ }^{4}$ Unit of Cancer Epidemiology, CPO-Piemonte and University of Turin, Turin 10126, Italy. \\ ${ }^{5}$ Escuela de Ciencias Biológicas, Universidad Pedagógica y Tecnológica de Colombia, Tunja 39-115, Colombia. \\ ${ }^{6}$ Department of Medical Sciences, University of Turin, Turin 10126, Italy. \\ ${ }^{7}$ Italian Institute for Genomic Medicine, Turin 10126, Italy. \\ ${ }^{8}$ Medical Genetics Unit, AOU Città della Salute e della Scienza, Turin 10126, Italy. \\ ${ }^{9}$ Department of Oncology, University of Turin, Turin 10126, Italy. \\ ${ }^{10}$ Molecular Genetics and Biology Unit, Santa Croce e Carle Hospital, Cuneo 12100, Italy. \\ ${ }^{11}$ Division of Medical Oncology, SS. Antonio e Biagio General Hospital, Alessandria 15121, Italy. \\ ${ }^{12}$ Pathology Unit, SS. Antonio e Biagio General Hospital, Alessandria 15121, Italy. \\ ${ }^{13}$ Department of Health Sciences, Section of Pathological Anatomy, University of Piemonte Orientale, Novara \\ 28100, Italy. \\ ${ }^{14}$ Clinical Genetics Unit, AO San Camillo-Forlanini, University La Sapienza, Rome 00185, Italy.
}


Introduction: Malignant Pleural Mesothelioma (MPM) is a rare, aggressive cancer caused by asbestos exposure. A genetic predisposition has been suggested to explain the occurrence of multiple cases in the same family and the observation that not all individuals highly exposed to asbestos develop the tumor. Germline variants in BAP1 are responsible for a rare cancer-prone syndrome (BAP1-TPDS) that includes mesothelioma in its cancer constellation. $C D K N 2 A$ and several DNA repair genes have been reported as further predisposing genes.

Results: We searched for BAP1 germline variants in 25 new probands with suspected BAP1-TPDS and we found a new pathogenic germline variant that affects splicing: $c .783+2 \mathrm{~T}>\mathrm{C}$. We calculated that the prevalence of the truncating variants in patients with familial MPM and MPM and other tumors is $7.7 \%(3 / 39)$. Cumulative asbestos exposure was assessed quantitatively in our cohort of patients to compare patients carrying pathogenic variants in $B A P_{1}, C D K N_{2} A$ and DNA repair genes $(n=14)$ to patients without variants in 94 cancer predisposing genes $(n=67)$. We showed that patients with variants in $B A P_{1}, C D K N_{2} A$ and DNA repair genes had a lower asbestos exposure than non-mutated patients $(P=0.00002)$.

Conclusion: Our results suggest that other genes could be involved in the genetic predisposition to mesothelioma. These data support the hypothesis of an increased asbestos sensitivity in patients with germline variants in $C D K N 2 A, B A P 1$ or DNA repair genes. According to the concept of BRCAness, patients with germline mutations in genes involved in homologous recombination repair may respond to drugs (e.g., PARP inhibitors) that induce synthetic lethality, similarly to patients with familial ovarian cancer and $B R C A 1 / B R C A 2$ inherited mutations.

\title{
27. Resveratrol reverts the EMT phenotype induced by lysophosphatidic acid in ovarian cancer cells through restoration of autophagy
}

\author{
Alessandra Ferraresi ${ }^{1}$, Christian Seca ${ }^{1}$, Chiara Vidoni $^{1}$, Carlo Girone ${ }^{1}$, Ji Hee $\mathrm{Ha}^{2}$, Danny N. \\ Dhanasekaran ${ }^{2}$, Ciro Isidoro ${ }^{1}$
}

${ }^{1}$ Laboratory of Molecular Pathology and Nanobioimaging, Department of Health Sciences, Università del Piemonte Orientale “A. Avogadro", Via Solaroli 17, Novara 28100, Italy.

${ }^{2}$ Stephenson Cancer Center and Department of Cell Biology, The University of Oklahoma Health Sciences Center, Oklahoma City, OK 73104, USA.

Introduction: Ovarian cancer emerges as a highly aggressive metastatic disease characterized by a high grade of lethality due to its asymptomatic nature and the late diagnosis, when cancer cells already spread in distant organs. Cancer progression is facilitated by pro-invasive factors, released by ovarian cancer cells and CAFs, that promote the Epithelial-to-Mesenchymal transition (EMT). Lysophosphatidic acid (LPA), a bioactive phospholipid abundantly present in ascitic fluid and plasma of ovarian cancer patients, stimulates the invasiveness of cancer cells. Resveratrol (RV), a natural-occuring polyphenol, is attracting the interest of many researchers due to its several anti-cancer properties. Of note, RV is a strong autophagy inducer.

Results: We found that LPA elicits cell migration in a panel of ovarian cancer cell models characterized by different grades of malignancy. In parallel, LPA induces EMT through the inhibition of autophagy in the cancer cells at the migration front. Interestingly, RV restores autophagy and halts ovarian cancer cell motility even in the presence of LPA.

Conclusion: Our findings indicate that restoration of autophagy by RV prevents LPA-induced tumor invasion and suggest that there is a functional cross-talk between autophagy and EMT phenotype. 


\title{
28. Spiperone, an antipsychotic, induces colorectal carcinoma cell death by a calcium- mediated apoptosis
}

\author{
Annamaria Antona ${ }^{1}$, Konkonika Roy ${ }^{1}$, Beatrice Riva ${ }^{2}$, Suresh Velnati ${ }^{1,3}$, Marco Varalda ${ }^{1}$, Gianluca \\ Baldanzi $^{1,3}$, Armando Genazzani $^{2}$, Daniela Capello ${ }^{1}$ \\ ${ }^{1}$ Dept Traslational Medicine, Università del Piemonte Orientale, Novara 28100, Italy. \\ ${ }^{2}$ Dept Pharmaceutical Sciences, Università del Piemonte Orientale, Novara 28100, Italy. \\ ${ }^{3}$ Center for translational research on Allergic and Autoimmune Diseases (CAAD), Novara 28100, Italy.
}

Colorectal cancer (CRC), one the most common malignancies, with increasing incidence and mortality rate worldwide, requires novel therapeutic strategies for its treatment. As developing new drugs is a timeand cost-intensive process with unexpected side effects, drug repositioning provides an effective strategy for accelerating drug development. Some antipsychotics currently used in human therapy reported potential anti-tumoral activity, thus in this study we intended to investigate their activity against CRC and CRC-stem cells (CRC-SCs).

We screened a panel of commercially available psychotropic drugs for their antitumoral activity on HCT116 cell line. Regardless for their receptor specificity, the viability data showed that this cell line was sensitive to just some drugs, especially spiperone, with an $\mathrm{IC}_{50}$ of $4 \mu \mathrm{mol} / \mathrm{L}$. Spiperone was also highly toxic for CRCSCs, inducing cell death by both apoptosis and necrosis with an $\mathrm{IC}_{50}<6 \mu \mathrm{mol} / \mathrm{L}$. To verify its mechanism of action, we investigated the role of spiperone on $\mathrm{Ca}^{2+}$ homeostasis in HCT116 by using the Fura-2 probe. We observed that it increases cytoplasmic $\mathrm{Ca}^{2+}$ in CRC cells in a dose dependent manner. Experiments performed in the absence of extracellular $\mathrm{Ca}^{2+}$ demonstrated that spiperone increased cytoplasmic $\mathrm{Ca}^{2+}$ originating from endoplasmic reticulum (ER); this was further confirmed by the induction of ER depletion with 2,5-tBHQ (specific SERCA inhibitor) followed by spiperone treatment resulted in an accentuated store operated $\mathrm{Ca}^{2+}$ entry response. Restoration of extracellular $\mathrm{Ca}^{2+}$ in the presence of spiperone further increased cytoplasmic $\mathrm{Ca}^{2+}$, suggesting that this drug is also active on plasma membrane $\mathrm{Ca}^{2+}$ channels.

Concluding, our data suggest that the anti-tumoral activity of spiperone might be due to $\mathrm{Ca}^{2+}$ movements through ER and plasma membrane channels. Further experiments are needed to better clarify the mechanism of action of spiperone in $\mathrm{Ca}^{2+}$ signalling and its possible repurposing as an antineoplastic drug.

\section{Circulating tumor cells in patients with non small cell lung cancer: pilot study, initial results}

\section{Roberta Carbone ${ }^{1}$, Sara Parini ${ }^{2}$, Marzia De Marni ${ }^{1}$, Silvia Restelli ${ }^{1}$, Paolo Aretini ${ }^{3}$, Francesca Lessi $^{3}$, Chiara Maria Mazzanti ${ }^{3}$, Renzo Luciano Boldorini ${ }^{4}$, Elena Trisolini ${ }^{4}$, Ciro Isidoro ${ }^{5}$, Caterina Casadio ${ }^{2}$}

\author{
${ }^{1}$ Tethis S.p.A. Via Russoli, 3, Milan 20143, Italy. \\ ${ }^{2}$ Thoracic Surgery department, Università del Piemonte Orientale, Novara 28100, Italy. \\ ${ }^{3}$ Fondazione Pisana Per la Scienza, S. Giuliano Terme, Pisa 56017, Italy. \\ ${ }^{4}$ SCDU Pathology department, Università del Piemonte Orientale, Novara 28100, Italy. \\ ${ }^{5}$ Department of Health Sciences, Università del Piemonte Orientale, Novara 28100, Italy.
}

Introduction: Lung cancer is the main cause of cancer mortality worldwide and the number of new cases is still rising. Early detection might be paramount to diagnose curable stages. There is growing interest about isolation of circulating tumor cells (CTC). This pilot study aimed at isolating epithelial putative CTCs in peripheral blood of early stage lung cancer patients using Smart BioSurface CTC assay, a nanoparticle- 
coated slide that quickly immobilizes living nucleated cells, thus avoiding pre-selection and any change in cell structure and biology.

Experimental model: Twenty-two patients undergoing surgical lung resection have been included in the preliminary study. Blood sample was collected from patients and the white blood cells fraction was let adhere on SBS-CTC slide. The cells were stained with anti-CD45 and anti-pan-CK antibodies. Positive cells (putative CTCs) were detected with an automated fluorescence microscope, isolated by laser capture microdissection and characterized for gene mutations.

Results: All patients were positive for at least 1 epithelial cell (putative CTC)/mL of blood, though no correlation between the number of epithelial cells and the stage of the disease was observed. NGS analysis revealed gene variants associated with tumors in all patients, of whom 13 patients had mutations in genes that are specifically associated with lung cancer. One patient carries BRAF V600E mutation, that has been identified also in the tissue biopsy.

Conclusion: Epithelial cells are present in the blood of lung cancer patients. Further analyses of the genotype and phenotype are in the process to determine whether these cells are indeed CTSs.

\section{Benefit sharing and globalisation of industry sponsored clinical trials for breast cancer research}

\section{Anil Babu Payedimarri, Gianluca Gaidano}

Department of Translational Medicine, University of Eastern Piedmont, Novara 28100, Italy.

Introduction: The burden of cancer is the greatest and rising most rapidly in low-income and middle-income countries (LMICs). The survival of breast cancer is especially poor in LMICs due to late stage presentation and inadequate access to therapy. Whereas low-income countries (LICs) suffer from a generalized lack of access to cancer care, in middle-income countries (MICs) such services and facilities may exist. However, highly-priced innovative medicines are often only affordable for certain subsets of the population, and good outcomes remain biased toward those who can pay. We carried out comprehensive analysis of the geographic distribution of breast cancer clinical trials involving at least one LMICs clinical site and trials evaluating costly innovative medicines.

Methodology: Data were extracted from clinicaltrials.gov registry (as of 30 Jun 2018). Advanced search filters used: (1) condition/disease: breast cancer; (2) study type: Interventional studies; (3) phases: I to IV; (4) funder type: industry. Countries were classified by income according to the World Bank (Fiscal year 2019).

Results: We analysed 1,746 trials. The fraction of phase III trials involving MICs sites was $55.36 \%$ (155/280) in which Lower-MICs (L-MICs) were 27.14\% (76/280) and Upper-MICs (UMICs) were 54.64\% (153/280). Smaller proportions of Phase I and II trials were conducted in MICs i.e. 5.92\% (26/439) and 16.23\% (161/992). Phase IV trials that involve MICs were $31.43 \%$ (11/35). No trials were conducted in LICs. L-MICs countries with the highest number of trials were India $(n=63)$ and Ukraine $(n=56)$ followed by Philippines $(n=23)$, Egypt $(n=17)$, and Pakistan $(n=10)$. For U-MICs, Russian Federation $(n=141)$, Brazil $(n=113)$, China $(n=$ $112)$, followed by Mexico $(n=88)$ and Turkey $(n=66)$.

Conclusion: Our analysis of the case of industry sponsored clinical trials with new, innovative medicines for breast cancer confirms previous observations with rarer blood cancers, that such trials are increasingly 
globalized, i.e., delocalized to countries that do not have the means to ensure (universal) access to high-cost medicines. Although substantial numbers of anti-cancer medicines are nowadays included in national lists of LMICs, their affordability and accessibility at country-level are often far from ensured. The relevance of benefit sharing in international research is still poorly understood. A legal framework formulating benefitsharing requirements in international research is necessary.

\title{
31. TP53 analysis in hematological malignancies
}

\section{Ahad A. Kodipad, Silvia Rasi, Clara Deambrogi, Simone Favini, Denise Peroni, Sruthi Sagiraju, Ramesh Adhinaveni, Sreekar Kogila, Fary Diop, Chiara Favini, Riccardo Moia, Lorenzo De Paoli, Gloria Margiotta Casaluci, Elena Crisà, Andrea Patriarca, Gianluca Gaidano}

Division of Hematology, Department of Translational Medicine, Amedeo Avogadro University of Eastern Piedmont, Novara 28100, Italy.

Introduction: Chronic lymphocytic leukemia (CLL) is the most common type of adult leukemia in the Western World. TP53 disruption (e.g., 17p del and/or TP53 mutations) is associated with chemorefractoriness and is now used in the clinical practice as a predictive biomarker. Moreover, also in myelodysplastic syndrome (MDS) TP53 seems to be associated with an inferior outcome. Based on these observations, the objective of the project is to characterize TP53 mutations in a prospective cohort of CLL and MDS patients to better define the frequency and the clinical implications of TP53 mutations.

Experimental model: The study is based on the analysis of 53 CLL and 26 MDS patients. The mutational analysis of the TP53 gene was performed using genomic DNA extracted from tumor cells. Peripheral blood was collected for CLL patients, while bone marrow for MDS patients. The TP53 gene was analyzed by Sanger sequencing from exons 2 to 11 .

Results: In the CLL group 5/53 (9.4\%) cases showed TP53 mutation. All mutations were missense and were reported in the IARC database. All TP53 mutations predicted functional consequences and were all reported as pathogenic mutations. At diagnosis, TP53 mutations associated with advanced age $(P=0.031)$, advanced Binet stage $(P=0.021)$ and higher $\beta 2$-microglobulin levels $(P=0.018)$. In MDS $2 / 26(7.7 \%)$ patients harbored TP53 mutation. These two mutations were missense and were validated by the IARC database. Both of TP53 mutated patients were treated with azacitidine but progressed early after treatment start.

Conclusion: In this prospective CLL cohort, TP53 mutations reflect the mutation profile already reported, with the novel finding of the correlation between TP53 and higher $\beta 2$-microglobulin levels. In MDS, TP53 seems to be associated with an advance disease and with early progression after therapy. Longer follow up is needed for univariate and multivariate survival analysis stratified according to TP53 mutations.

\section{Epigenetic changes in ovarian cancer cells subjected to starvation or to the caloric restriction mimetic Resveratrol}

\author{
Letizia Vallino ${ }^{1}$, Alessandra Ferraresi ${ }^{1}$, Chiara Vidoni ${ }^{1}$, Claudia Lora, Giovanna Chiorino ${ }^{2}$, \\ Alessandra Galetto $^{3}$, Ciro Isidoro ${ }^{1}$
}

${ }^{1}$ Laboratory of Molecular Pathology, Department of Health Sciences, Università del Piemonte Orientale "A. Avogadro", Via Solaroli 17, Novara 28100, Italy. 
${ }^{2}$ Cancer Genomics Laboratory, Fondazione Edo ed Elvo Tempia, Biella 13900, Italy.

${ }^{3}$ Oncology Unit, Department of Translational Medicine, Università del Piemonte Orientale "A. Avogadro", Novara 28100, Italy.

Introduction: Biological processes are regulated through epigenetics, including chromatin remodelling (methylation, acetylation, etc.) and non coding RNAs. The correct balance of these mechanisms is crucial for the maintenance of cellular homeostasis and its dysregulation is correlated to many disorders such as cancer. Nutrient availability has a great impact on the metabolic pathways involved in cancer cell proliferation. Incubation in Earle's Balanced Salt Solution (EBSS), a culture medium containing 1\% glucose in absence of serum and amino acids, mimics the condition of starvation. Resveratrol (RV), a dietary polyphenol acting as a protein (caloric) restriction mimetic makes the cells unable to uptake nutrients for their metabolism.

Results: Here, we report on the changes of the miRNAs in ovarian cancer cells subjected to amino acid starvation or to RV. In this fasting condition, cancer cells promote autophagy as a pro-survival mechanism and eventually exit the cell cycle to undergo a dormant state. RV has a major impact on miRNome involved in autophagy and apoptosis compared to the one observed in EBSS-treated cells.

Conclusion: Our data support the view that RV treatment can be more effective than nutrient starvation, thus it can substitute it in order to induce a dormant-like state in cancer cells.

\section{KMT2D mutations and TP53 disruptions are poor prognostic biomarkers in MCL receiving} high-dose therapy: a fil study

Chiara Favini ${ }^{1}$, Riccardo Moia ${ }^{1}$, Fary Diop ${ }^{1}$, Simone Ferrero ${ }^{2,3}$, Davide Rossi ${ }^{4,5}$, Andrea Rinaldi ${ }^{5}$, Alessio Bruscaggin ${ }^{5}$, Valeria Spina ${ }^{5}$, Andrea Evangelista ${ }^{6}$, Ivo Kwee ${ }^{5,7,8}$, Alice Di Rocco ${ }^{9}$, Vittorio Stefoni $^{10}$, Paola Ghione ${ }^{2}$, Daniela Barbero ${ }^{2}$, Domenico Novero ${ }^{11}$, Marco Paulli ${ }^{12}$, Alberto Zamò ${ }^{13,14}$, Maria Gomes da Silva ${ }^{15}$, Armando Santoro ${ }^{16}$, Annalia Molinari ${ }^{17}$, Andres Ferreri ${ }^{18}$, Andrea Piccin $^{19}$, Sergio Cortelazzo ${ }^{20}$, Francesco Bertoni ${ }^{5}$, Marco Ladetto ${ }^{21}$, Gianluca Gaidano ${ }^{1}$

\footnotetext{
${ }^{1}$ Division of Hematology, Department of Translational Medicine, University of Eastern Piedmont, Novara 28100, Italy.

${ }^{2}$ Department of Molecular Biotechnologies and Health Sciences - Hematology Division, Università di Torino, Torino 10126, Italy.

${ }^{3}$ Hematology Division, AOU Città della Salute e della Scienza di Torino, Torino 10126, Italy.

${ }^{4}$ Hematology, Oncology Institute of Southern Switzerland, Bellinzona 6500, Switzerland.

${ }^{5}$ Universita' della Svizzera italiana, Institute of Oncology Research, Bellinzona 6500, Switzerland.

${ }^{6}$ Clinical Epidemiology, Città della Salute e della Scienza and CPO Piemonte, Torino 10126, Italy.

${ }^{7}$ Swiss Institute of Bioinformatics (SIB), Lausanne 1015, Switzerland.

${ }^{8}$ Dalle Molle Institute for Artificial Intelligence (IDSIA), Manno CH-6928, Switzerland.

${ }^{9}$ Department of Cellular Biotechnologies and Hematology, Policlinico Umberto I, "Sapienza" University of Rome, Roma 00185, Italy.

${ }^{10}$ Institute of Hematology "L. e A. Seràgnoli”, University of Bologna, Bologna 40126, Italy.

${ }^{11}$ First unit of Pathology, AOU Città della Salute e della Scienza di Torino, Torino 10126, Italy.

${ }^{12}$ Unit of Anatomic Pathology, Department of Molecular Medicine, Fondazione IRCCS Policlinico San Matteo and Università degli Studi di Pavia, Pavia 27100, Italy.

${ }^{13}$ Department of Oncology, Università di Torino, Torino 10126, Italy.

${ }^{14}$ Department of Diagnostics and Public Health, University of Verona, Verona 37129, Italy.

${ }^{15}$ Department of Hematology, Instituto Português de Oncologia de Lisboa, Lisboa 1099-023, Portugal.
} 
${ }^{16}$ Humanitas Cancer Center, Humanitas Clinical and Research Center, Rozzano 20089, Italy.

${ }^{17}$ Hematology, Ospedale degli Infermi, Rimini 47923, Italy.

${ }^{18}$ Lymphoma Unit, Department of Onco-Haematology, IRCCS San Raffaele Scientific Institute, Milano 20132, Italy.

${ }^{19}$ Department of Hematology, Ospedale Generale, Bolzano 39100, Italy.

${ }^{20}$ Oncology Unit, Humanitas/Gavazzeni Clinic, Bergamo 24125, Italy.

${ }^{21}$ SC Ematologia, Azienda Ospedaliera Santi Antonio e Biagio e Cesare Arrigo, Alessandria 15121, Italy.

Introduction: In recent years, the outcome of mantle cell lymphoma (MCL) has improved, especially in younger patients, receiving cytarabine-containing chemoimmunotherapy and autologous stem cell transplantation. Nevertheless, a proportion of MCL patients still experience early failure. The aims of the study are to identify biomarkers anticipating failure of intensive chemotherapy in MCL.

Experimental model: We performed target resequencing and DNA profiling of purified tumor samples collected from patients enrolled in the prospective FIL-MCL0208 phase III trial (NCT02354313, high-dose chemoimmunotherapy followed by autologous transplantation and randomized lenalidomide maintenance).

Results: Mutations of KMT2D and disruption of TP53 by deletion or mutation associated with an increased risk of progression and death, both in univariate and multivariate analysis. By adding KMT2D mutations and TP53 disruption to the MIPI-c backbone, we derived a new prognostic index, the "MIPI-genetic". The "MIPI-g" improved the model discrimination ability compared to the MIPI-c alone, defining three risk groups: (1) low-risk patients (4-year PFS and OS of 72.0\% and 94.5\%); (2) intermediate-risk patients (4-year PFS and OS of $42.2 \%$ and $65.8 \%$ ); and (3) high-risk patients (4-year PFS and OS of $11.5 \%$ and $44.9 \%$ ).

Conclusion: Our results: (1) confirm that TP53 disruption identifies a high-risk population characterized by poor sensitivity to conventional or intensified chemotherapy; (2) provide the pivotal evidence that patients harboring KMT2D mutations share the same poor outcome as patients harboring TP53 disruption; and (3) allow to develop a tool for the identification of high-risk MCL patients for whom novel therapeutic strategies need to be investigated.

\section{In vitro and in vivo activity of biomimetic magnetic nanoparticles for drug delivery in presence of gradient magnetic field}

\section{Maria Prat ${ }^{1}$, Francesca Oltolina ${ }^{1}$, Ana Peigneux Navarro ${ }^{2}$, Donato Colangelo ${ }^{1}$, Concepcion Jimenez-Lopez ${ }^{2}$}

${ }^{1}$ Laboratory of Histology, Department of Health Sciences, Università del Piemonte Orientale "A. Avogadro", Novara 28100, Italy.

${ }^{2}$ Departamento de Microbiología, Facultad de Ciencias, Universidad de Granada, Fuentenueva, Granada 18071, Spain.

Nanotechnology and nanoparticles (NPs) have become very attractive for their applications in different fields, comprising biology, medicine and oncology. In this context, magnetite nanoparticles are even more interesting as they can be manipulated by an external magnetic field, besides being multifunctional platforms.

Herein, we describe a drug delivery system based on biomimetic magnetic nanoparticles (BMNPs) synthetized in presence of MamC protein from Magnetococcus marinus MC-1. MamC controls the 
morphology and size of the crystals $(\sim 40 \mathrm{~nm})$, that are paramagnetic at room and body temperature. Because of this protein, BMNPs have a negative surface charge at physiological $\mathrm{pH}$ values that allow an efficient coupling with different molecules.

These BMNPs were functionalized with the chemotherapy drug doxorubicin (DOXO) and their ability to respond to an externally applied gradient magnetic field (GMF) was studied both in vitro and in vivo. Naive BMNPs were cytocompatibles on $4 \mathrm{~T} 1$ cells, while the DOXO-BMNPs were toxic starting from short exposure times when a GMF is applied. This allowed a faster interaction between BMNPs and cells (Perls Blue Staining) as well as a faster delivery of the DOXO to cell nuclei (confocal analysis). When DOXOfunctionalized or not functionalized BMNPs were i.v. injected in mice bearing 4T1 cells-induced tumors, the application of the magnet on the tumors for $1 \mathrm{~h}$ reduced their size. Moreover, the presence of DOXO on BMNPs enhanced this effect.

All together, these data suggest that tumor attack by combined strategies (chemotherapeutic drug and magnetic field) could represent a promising approach for cancer therapy. Future steps will be the possibility to apply an alternating magnetic field to BMNPs to induce hyperthermia, to which tumor cells are more sensitive than healthy cells, and to enhance a more efficient localized release of the drug at the tumor site.

\section{Novel diacylglycerol kinase alpha inhibitors for X-linked lymphoproliferative disease 1 therapy}

\section{Suresh Velnati ${ }^{1,2}$, Elisa Ruffo ${ }^{1,3}$, Alberto Massarotti $^{4}$, Maria Talmon ${ }^{5}$, Sai Sandeep Varma Konduru $^{1,2}$, Annamaria Antona ${ }^{1}$, Alessandro Gesu', Luigia Grazia Fresu ${ }^{5}$, Andrew L Snow ${ }^{6}$, Daniela Capello ${ }^{1}$, Alessandra Bertoni ${ }^{1}$, Gian Cesare Tron $^{4}$, Andrea Graziani ${ }^{1,3}$, Gianluca Baldanzi ${ }^{1,2}$}

\footnotetext{
${ }^{1}$ Department of Translational Medicine, University of Piemonte Orientale, via Solaroli 17, Novara 28100, Italy. ${ }^{2}$ Center for translational research on Allergy and Autoimmune Diseases (CAAD), Novara 28100, Italy. ${ }^{3}$ Division of Experimental Oncology, School of Medicine, University Vita e Salute San Raffaele, Milan 20132, Italy.

${ }^{4}$ Department of Pharmaceutical Sciences, University of Piemonte Orientale, largo Donegani 12, Novara 28100, Italy.

${ }^{5}$ Department of Health Sciences, School of Medicine, University of Piemonte Orientale, Novara 28100, Italy. ${ }^{6}$ Department of Pharmacology and Molecular Therapeutics, Uniformed Services University of the Health Sciences, Bethesda, MD 20814, USA.
}

Background: X-linked lymphoproliferative disease 1 (XLP1) is a primary immunodeficiency due to mutations in the SH2D1a gene, encoding SAP. SAP deficiency perturbs TCR signalling and results in a constitutive diacylglycerol kinase alpha (DGK $\alpha$ ) activity that impairs CD8+ T cell restimulation induced cell death (RICD). Indeed, pharmacological inhibition of DGK $\alpha$ in XLP1 animal models limits CD8+ T cell expansion and interferon- $\gamma$ production, suggesting the development of DGK $\alpha$ inhibitors for XLP1 therapy.

Experimental model: To find new DGK $\alpha$ inhibitors, we used a 2D/3D in silico approach based on chemical homology with the two commercially available DGK $\alpha$ inhibitors (R59922 and R59949). The library was screened for inhibitory activity at $100 \mu \mathrm{mol} / \mathrm{L}$. Active compounds were tested at concentration from 0.1 to $100 \mu \mathrm{mol} / \mathrm{L}$ to estimate the $\mathrm{IC}_{50}$. Furthermore, we tested the most active compounds in RICD assay using SAP silenced lymphocytes as XLP1 model. 
Results: Out of the resulting 127 compounds, ritanserin (serotonin antagonist) and compoundo1 (uncharacterized molecule) were highly specific for DGK $\alpha$ and showed superior potency compared to R59022 and R59949. In cellular models of XLP-1, both ritanserin and compound01 restored RICD of SAPdeficient CD8+ without significant toxicity. Moreover, compound01 doesn't perturb serotonin signalling. Thus, we executed compound optimization of compound01 that yielded compound02-07 (synthesized compounds). All those compoundo1 derivatives are highly specific to DGK $\alpha$ without perturbing serotonin signalling.

Conclusion: Concluding our work allows us to propose a pharmacological model for the rational design of DGK $\alpha$ inhibitors and may contribute to the development of innovative therapies for diseases characterized by RICD resistance such as XLP-1. 